Luísa Grilo de Abreu

\title{
EVOLUÇÃO DO PADRÃO DE CONSUMO DE FAMÍLIAS DE BAIXA RENDA NO RIO DE JANEIRO
}

Dissertação de Mestrado

Dissertação apresentada ao Programa de Pósgraduação em Administração de Empresas da PUCRio como requisito parcial para obtenção do título de Mestre em Administração de Empresas.

Orientador: Prof. Luis Fernando Hor-Meyll Alvares 
Luísa Grilo de Abreu

Evolução do padrão de consumo de famílias de baixa renda no Rio de Janeiro

Dissertação apresentada como requisito parcial para obtenção do grau de Mestre pelo Programa de Pósgraduação em Administração de Empresas da PUC-Rio. Aprovada pela Comissão Examinadora abaixo assinada.

Prof. Luis Fernando Hor-Meyll Alvares

Orientador Departamento de Administração - PUC - Rio

Prof. Paulo Cesar de Mendonça Motta Departamento de Administração - PUC - Rio

Prof. Valdecy Leite

UFRJ

Profa. Mônica Herz

Vice-Decana de Pós-Graduação do CCS

Rio de Janeiro, 29 de agosto de 2011 
Todos os direitos reservados. É proibida a reprodução total ou parcial do trabalho sem autorização da universidade, da autora e do orientador.

\section{Luísa Grilo de Abreu}

Graduou-se em Estatística pela ENCE (Escola Nacional de Ciências Estatísticas) em 2007. Trabalha no IBGE desde 2010.

Ficha Catalográfica

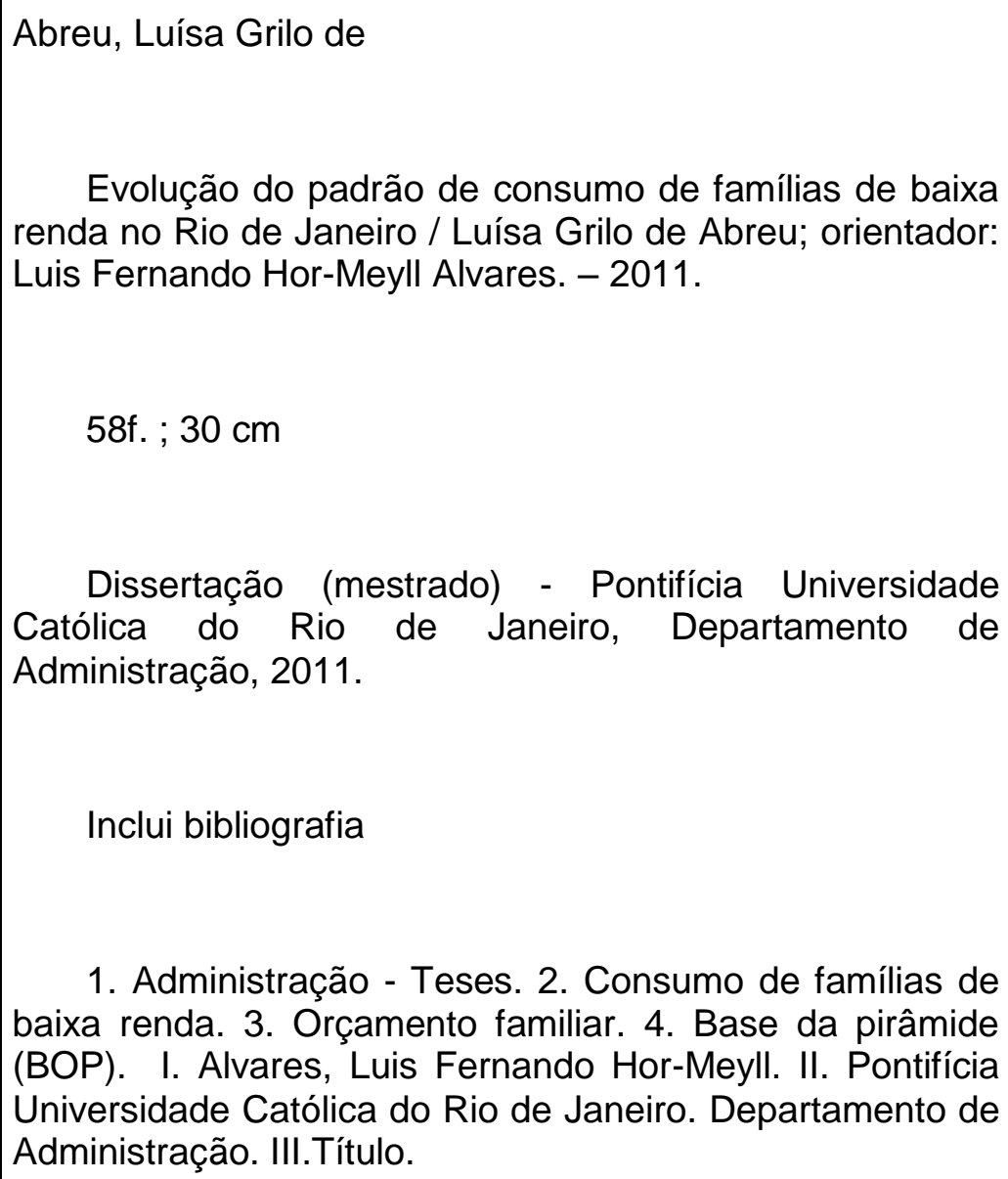

Evolução do padrão de consumo de famílias de baixa renda no Rio de Janeiro / Luísa Grilo de Abreu; orientador: Luis Fernando Hor-Meyll Alvares. - 2011.

58f. ; $30 \mathrm{~cm}$

Dissertação (mestrado) - Pontifícia Universidade Católica do Rio de Janeiro, Departamento de Administração, 2011.

Inclui bibliografia

1. Administração - Teses. 2. Consumo de famílias de baixa renda. 3. Orçamento familiar. 4. Base da pirâmide (BOP). I. Alvares, Luis Fernando Hor-Meyll. II. Pontifícia Universidade Católica do Rio de Janeiro. Departamento de Administração. III.Título. 
Aos meus pais, Grace e Marcos, por todo amor, apoio e confiança. 


\section{Agradecimentos}

Aos meus pais, Grace e Marcos, por todo o amor e por tudo que me ensinaram.

A Maureen e Jiro, pelo carinho, apoio e incentivo.

Ao meu namorado, Felipe, pelo carinho, força e por sempre estar ao meu lado.

Ao meu orientador, professor Luis Fernando Hor-Meyll, por todo apoio e ensinamentos.

Ao professor Marcelo Maia pela ajuda e incentivo.

Aos colegas e profissionais do IBGE que muito me ajudaram no trabalho com a POF

A todos aqueles que de alguma forma contribuíram para a execução deste trabalho e conclusão de mais uma importante etapa da minha vida. 


\section{Resumo}

Abreu, Luísa Grilo de; Alvares, Luis Fernando Hor-Meyll. Evolução do Padrão de Consumo de Famílias de Baixa Renda no Rio de Janeiro. Rio de Janeiro, 2011. 58p Dissertação de Mestrado - Departamento de Administração, Pontifícia Universidade Católica do Rio de Janeiro.

Existem, na literatura de Marketing no Brasil, poucos estudos de natureza quantitativa sobre o orçamento das famílias de baixa renda, destacando-se o de Silva e Parente (2007). Entretanto, estudos que considerassem um período em que efeitos do aumento real do salário mínimo, a oferta de crédito e programas sociais recentemente implantados pelo governo brasileiro sobre a forma como essas famílias alocam seu orçamento não foram encontrados. O objetivo desta dissertação foi identificar padrões de consumo de famílias de baixa renda, da região metropolitana do Rio de Janeiro, a partir da composição de seu orçamento, nos anos de 2002/2003 e 2008/2009, e analisar mudanças encontradas nesse período. Com base nos dados fornecidos pela Pesquisa de Orçamentos Familiares (POF/IBGE) e utilizando técnica de análise de grupamentos (cluster), foram identificados os mesmos quatro perfis de consumo nos dois períodos estudados, o que parece confirmar a heterogeneidade do segmento de baixa renda. Verificou-se que os perfis não apresentaram sensíveis mudanças no intervalo estudado, embora tenham surgido evidências de melhora do padrão geral de consumo das famílias de baixa renda e de sua qualidade de vida.

\section{Palavras-chave}

Consumo de famílias de baixa renda; orçamento familiar; base da pirâmide (BOP) 


\section{Abstract}

Abreu, Luísa Grilo de; Alvares, Luis Fernando Hor-Meyll (Advisor). Evolution of the Consumption Pattern of Low-Income Families in Rio de Janeiro. Rio de Janeiro, 2011. 58p MSc. Dissertation - Departamento de Administração, Pontifícia Universidade Católica do Rio de Janeiro.

There are, in the brazilian marketing literature, few quantitative studies about the low-income families budget, especially that of Silva and Parente (2007). However, studies that consider a period in which the effects of rising real minimum wage, the supply of credit and social programs recently implemented by the Brazilian government on how these families allocate their budgets were not found. The goal of this dissertation was to identify patterns of low-income families from the metropolitan area of Rio de Janeiro, from the composition of its budget in the years 2002/2003 and 2008/2009, and analyze changes found in this period. Based on data from the Pesquisa de Orçamentos Familiares (POF / IBGE) and using cluster analysis, were identified the same four profiles of consumption in both periods studied, which seems to confirm the heterogeneity of the low income segment. It was found that the profiles did not show sensitive changes in the interval studied, although there have been evidence of improvement of the general pattern of consumption of low-income families and their quality of life.

\section{Keywords}

Consumption of low-income families; family budget; base-of-the-pyramid (BOP) 


\section{Sumário}

1 Introdução 11

1.1. Objetivo do Estudo 13

1.2. Relevância do Estudo 13

1.3. Delimitação do Estudo 13

$\begin{array}{ll}\text { 1.4. Pergunta de Pesquisa } & 14\end{array}$

1.5. Estrutura da Dissertação 14

2 Revisão Bibliográfica 15

2.1. Consumidor de Baixa Renda 15

2.2. Segmentação e Baixa Renda no Brasil 18

2.3. Orçamento Familiar 24

3 Método 26

3.1. Pesquisa de Orçamentos Familiares (POF) 26

3.2. Amostra 27

3.3. Análise de Cluster 28

3.3.1. Método Hierárquico 30

3.3.2. Método Não-Hierárquico 31

3.4. Aplicação da Análise de Cluster 32

4 Discussão dos Resultados 35

4.1. Descrição dos Segmentos $\quad 37$

$\begin{array}{ll}\text { 4.1.1. } 2002 / 2003 & 37\end{array}$

4.1.2. $2008 / 2009 \quad 40$

4.2. Comparação entre as situações das famílias em 2002/2003 e 2008/2009 43

4.3. Evolução entre 2002/2003 e 2008/2009 48

5 Considerações Finais $\quad 52$

5.1. Sugestões para trabalhos futuros 53

6 Referências Bibliográficas $\quad 55$ 


\section{Lista de figuras}

Figura 1: Reajustes do salário mínimo e evolução da inflação anual .................12

Figura 2: Percentual total de gastos por categorias de despesa .......................43 43

Figura 3: Quantidade de Alimento.............................................................. 44

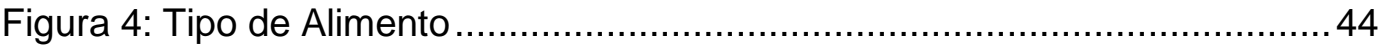

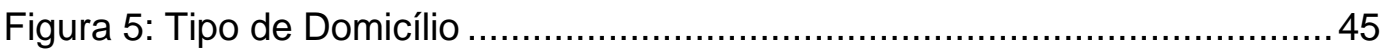

Figura 6: Condição de ocupação do domicílio............................................... 45

Figura 7: Sexo do chefe de domicílio ................................................................4 46

Figura 8: Posse de cartão de crédito ............................................................ 46

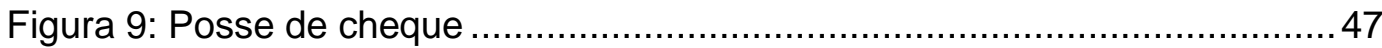

Figura 10: Avaliação da condição de vida ................................................. 47

Figura 11: Percentual de famílias com dívidas ...............................................48

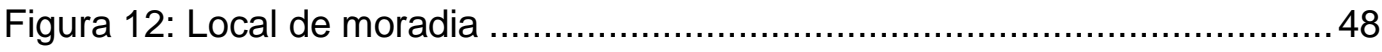

Figura 13: Novas concessões de crédito - pessoa física ................................50 


\section{Lista de tabelas}

Tabela 1: Domicílios particulares, por classes de rendimento domiciliar mensal (PNAD, 2009)................................................................................ 11

Tabela 2: Resumo do Orçamento Familiar dos clusters POF02/03 ..................... 35

Tabela 3: Resumo do Orçamento Familiar dos clusters POF08/09 .................... 36

Tabela 4: Renda familiar, número de moradores, idade e anos de estudo do chefe de família...................................................................................... 43

Tabela 5: Percentual de famílias por segmento ............................................49 